\title{
Wer sind die Aufsteiger, wer die Absteiger im deut- schen Bildungssystem? Eine Analyse auf Basis einer deutschlandweit repräsentativen Befragung und beson- derer Berücksichtigung des Geschlechts
}

\author{
Dirk Baier, Christian Pfeiffer
}

\section{Einleitung}

Im Jahr 2012 hat die Bertelsmann-Stiftung einen Bericht über den Aufbzw. Abstieg im deutschen Bildungssystem bezogen auf die Sekundarstufe I veröffentlicht (Bellenberg, 2012). In dem Text wird erstmals systematisch für die einzelnen Bundesländer der Schulwechsel dargestellt. Dass es sich dabei um ein relevantes Thema handelt, belegt allein die Zahl, dass davon jährlich ca. 100,000 Schüler betroffen sind. Nur in einem Viertel der Fälle handelt es sich dabei um einen Aufstieg (27.4\%), in 58.4\% der Fälle um einen Abstieg (Bellenberg, 2012, S. 10); 14.1\% der Schulformwechsler sind sog. „Umsteiger“, d. h. Schüler, die in einer Schulform, die mehrere Bildungsgänge anbietet, zwischen den Zweigen wechseln (z. B. in Gesamtschule zwischen Realschul- und Abiturzweig).

Die Wahrscheinlichkeit, einen Auf- oder Abstieg zu erleben, ist eng mit dem Bundesland und damit letztlich auch dem Schulsystem verknüpft. Wie die nachfolgende Tabelle 1 zeigt, ergeben sich hier beträchtliche regionale Unterschiede. Zum einen variiert der Anteil an Schulformwechslern zwischen den Bundesländern zwischen 1.3 und 6.1\%. Zum anderen findet sich, dass in Niedersachsen auf einen Schulaufstieg zehn Abstiege kommen. In Bayern hingegen sind die Aufstiege sogar etwas häufiger als die Abstiege.

Der Bericht der Bertelsmann-Stiftung konzentriert sich in der Analyse der Auf- und Abstiegsprozesse weitestgehend auf die Frage des Einflusses des Schulsystems. Diese Perspektive ist wichtig. Ergänzend erscheint es allerdings auch sinnvoll, die individuellen Merkmale der Schüler in die Betrachtung einzubeziehen. Hierzu möchten wir nachfolgend eine deutschlandweite Repräsentativbefragung heranziehen, die in den Jahren 2007 und 2008 vom Kriminologischen Forschungsinstitut Niedersachsen durchgeführt wurde (vgl. Baier, Pfeiffer, Rabold, Simonson \& Kappes, 
2010; Baier, Pfeiffer, Simonson \& Rabold, 2009). Anliegen dieses Beitrages ist es, ausgewählte individuelle Merkmale von Auf- und Absteigern gegenüber zu stellen und dabei die Frage zu beantworten, inwieweit sich diese beiden Gruppen sowohl untereinander als auch im Vergleich zu Jugendlichen, die keinen Schulwechsel erleben, unterscheiden. Besonderer Fokus wird dabei auf mögliche Geschlechterunterschiede gelegt. Der Beitrag hat einen explorativen Charakter, da es bislang wenig Forschung über die individuellen Merkmale von Auf- bzw. Absteigern gibt.

Tabelle 1. Anteil Schulformwechsler bzw. Verhältnis Auf- zu Abstieg nach Bundesland (Quelle: Bellenberg, 2012, S. 11, 41, 45 und 49; für Hamburg und Rheinland-Pfalz liegen keine Angaben vor).

\begin{tabular}{lcc}
\hline & $\begin{array}{c}\text { Anteil Schulform- } \\
\text { wechsler } \\
\text { in \% }\end{array}$ & $\begin{array}{c}\text { Verhältnis } \\
\text { Auf- zu Abstieg }\end{array}$ \\
\hline Baden-Württemberg & 1.3 & $1: 1.5$ \\
Bayern & 4.3 & $1: 0.9$ \\
Berlin & 4.9 & $1: 6.9$ \\
Brandenburg & 2.7 & $1: 2.5$ \\
Bremen & 6.1 & $1: 2.4$ \\
Hessen & 2.9 & $1: 8.7$ \\
Mecklenburg-Vorpommern & 2.9 & $1: 1.8$ \\
Niedersachsen & 2.6 & $1: 10.0$ \\
Nordrhein-Westfalen & 1.8 & $1: 5.6$ \\
Saarland & 3.3 & $1: 4.6$ \\
Sachsen & 1.6 & $1: 4.7$ \\
Sachsen-Anhalt & 1.4 & $1: 3.6$ \\
Schleswig-Holstein & 2.1 & $1: 3.9$ \\
Thüringen & 2.2 & $1: 3.2$ \\
\hline
\end{tabular}

Die vorhandenen Studien widmen sich primär der Frage, inwieweit der Schulformwechsel sozialstrukturell bedingt ist. Henz (1997) berichtet, dass der Anteil an Schülern, die die Schule wechseln, über die Kohorten hinweg von 5 auf 11.5\% gestiegen ist. Dabei hat sich die Wahrscheinlichkeit von Abstiegen deutlich erhöht, so dass Auf- und Abstiege mittlerweile gleich häufig vorkommen. Entscheidend ist, dass Schulformwechsel in sozial selektiver Weise vonstattengehen. Kinder aus besser gebildetem El- 
ternhaus steigen eher auf, Kinder aus schlechter gebildetem Elternhaus eher ab. Dies bestätigen auch die Auswertungen von Glaesser und Lauterbach (2006). Diese beschränken sich aber nicht auf die Untersuchung struktureller Variablen, sondern beziehen auch individuelle Faktoren mit ein. Zwei Befunde erscheinen dahingehend interessant: Erstens ergeben sich Geschlechterunterschiede, nach denen Frauen häufiger auf- und absteigen. Zweitens erhöht tägliches Rauchen das Risiko des Abstiegs. Auch die individuelle Leistungsbereitschaft hat einen Einfluss auf Schulwechselprozesse. Dies stützt die hier gewählte Perspektive, individuelle Faktoren in die Betrachtung einzubeziehen. Die individuellen Deutungsmuster und Verarbeitungsstrategien von Schulwechslern stehen zudem in der qualitativen Studie von Liegmann (2008) im Vordergrund. Anhand von Interviews mit Auf- und Absteigern in der sechsten und siebten Jahrgangsstufe wird gezeigt, dass es verschiedene Gruppen an ab- und aufsteigenden Schülern gibt. Je nachdem, zu welcher Gruppe ein Schüler gehört, bedarf es unterschiedlicher Formen der pädagogischen Begleitung des Schulwechsels. In der Studie wird damit die interne Heterogenität der Auf- und Absteiger illustriert. Nachfolgend wird allerdings kein gruppierendes Vorgehen zur Analyse des Schulformwechsels herangezogen, sondern ein variablenorientiertes Vorgehen.

\section{Die Befragung}

Die Schülerbefragung 2007/2008 wurde in 61 Landkreisen bzw. kreisfreien Städten Deutschlands durchgeführt. Sie widmete sich primär der Frage, in welchem Ausmaß und aus welchen Gründen Jugendliche Opfer und Täter von Gewalt geworden sind. Die Verteilung auf zwei Erhebungsjahre war notwendig, weil in einigen Bundesländern die Kompromissfindung zur Wahrung des Datenschutzes mehr Zeit in Anspruch nahm als in anderen Bundesländern. Die Gebiete wurden per Zufall derart bestimmt, dass die Stichprobe Repräsentativität für die gesamte Bundesrepublik Deutschland beansprucht und dass Auswertungen für verschiedene Regionen (Ost/West, Nord/Süd, Stadt/Land) möglich sind. Methodisch wurde der Weg der schulklassenbasierten Befragung gewählt. Die Befragungen wurden in den Gebieten in ca. jeder zweiten bzw. (in Großstädten) jeder sechsten zufällig ausgewählten Schulklasse durchgeführt, jeweils im Klassenverband und in Gegenwart eines Lehrers und eines eigens geschulten Testleiters. Befragt wurden dabei nur Klassen der neunten Jahrgangsstufe, da diese aufgrund ihres Alters am besten geeignet erscheint, um 
kriminalitätsbezogene Fragestellungen im Jugendalter zu untersuchen. In jüngeren Altersstufen sind die entsprechenden Verhaltensweisen weniger verbreitet. In älteren Altersstufen sind zwar teilweise höhere Raten der Viktimisierung oder der Täterschaft zu erwarten. Eine repräsentative Studie wäre mit ihnen aber über ein schulbasiertes Vorgehen nicht realisierbar, da ein Teil der Schüler ab der zehnten Jahrgangsstufe nicht mehr in Schulen zu erreichen ist.

Insgesamt wurden im Rahmen dieser Schülerbefragung 44,610 Jugendliche befragt, wobei eine Rücklaufquote von $62.1 \%$ erreicht wurde. In Großstädten wie im Ostteil Deutschlands fiel die Rücklaufquote unterdurchschnittlich aus. Etwa die Hälfte der befragten Jugendlichen ist männlich (51.3\%), die andere Hälfte weiblich. Das Durchschnittsalter der Befragten beträgt 15.3 Jahre. Etwa jeder vierte Befragte (27.4\%) hat einen Migrationshintergrund; die beiden größten Migrantengruppen sind türkischstämmige Jugendliche und Jugendliche, deren Familien aus Ländern der ehemaligen SU zugewandert sind (inkl. Aussiedler). Zur Bestimmung des Migrationshintergrundes wurden Angaben zum Geburtsland und zur Staatsangehörigkeit des Befragten sowie des leiblichen Vaters und der leiblichen Mutter herangezogen. In der Stichprobe befinden sich Schüler aller Schulformen, wobei auch Schulen in freier Trägerschaft einbezogen wurden. Etwa ein Viertel der Befragten besucht eine Förder- oder Hauptschule (26.8\%), etwas mehr ein Gymnasium (29.8\%). Die restlichen Schüler werden an Real- oder Gesamtschulen unterrichtet (43.4\%). Nicht in allen Gebieten stellt die realisierte Stichprobe ein genaues Abbild der Grundgesamtheit dar, wenn die Verteilung über die Schulformen betrachtet wird. Aus diesem Grund wurde ein Gewichtungsfaktor gebildet, der die Repräsentativität der Befragung für die einzelnen Gebiete und für Gesamtdeutschland sicher stellt (vgl. auch Baier et al., 2009). Alle hier präsentierten Auswertungen erfolgen anhand gewichteter Daten.

\section{Ergebnisse}

\subsection{Erfassung des Schulwechsels}

Die Schüler wurden gefragt, ob sie schon einmal einen Schulwechsel erlebt haben. Als Antwortvorgaben wurden folgende vier Kategorien angeboten, mit der Möglichkeit der Mehrfachantwort: Wechsel von höherer auf niedrigere Schulform (z. B. Gymnasium auf Realschule), Wechsel von niedriger auf höhere Schulform (z. B. Haupt- auf Realschule), anderer 
Wechsel (z. B. innerhalb der gleichen Schulform wegen Umzugs), keinen Schulwechsel.

Insgesamt 1,990 Schüler machten zu dieser Frage keine Angabe, 26,355 Schüler (62.2\% bezogen auf alle Befragten mit gültiger Angabe) gaben an, dass sie keinen Wechsel erlebt hätten. Von den verbleibenden Schülern (37.8\%) wurden in Bezug auf die Art des Wechsels z. T. Mehrfachnennungen abgegeben. Um in den nachfolgenden Auswertungen eindeutig Auf- und Absteiger unterscheiden zu können, wurden die Befragten wie folgt gruppiert: Befragte, die nur von einem Abstieg berichteten und sonst von keinem Wechsel, werden als „Absteiger“ klassifiziert, Befragte, die nur von einem Aufstieg berichten, als „Aufsteiger“. Wenn ein Befragter verschiedene Wechsel bzw. andere Wechsel erlebt hat, wird er zur ,sonstigen" Gruppe zugeordnet. Zusätzlich wurden die Angaben der Schüler derart bereinigt, dass Schüler aus Förderschulen keinen Abstieg, Schüler aus Gymnasien keinen Aufstieg erfahren haben können. Insgesamt wurden 251 Fälle, die möglicherweise irrtümlich an der entsprechenden Stelle ein Kreuz setzten, zu Fällen mit fehlenden Angaben eingestuft. Über diese Vorgehensweise konnten letztlich 10.2\% aller Schüler als Absteiger, 7.7\% als Aufsteiger und $19.9 \%$ als sonstige Wechsler kategorisiert werden. In der Kategorie der sonstigen Wechsler befinden sich auch Auf- oder Absteiger, bei denen gleichzeitig aber auch ein anderer Wechsel vorgelegen hat; ,sonstiger Wechsel“ umfasst damit auch mehrere Wechsel.

\subsection{Soziodemographische Charakterisierung der Auf- und Absteiger}

In Abbildung 1 ist dargestellt, wie sich die unterschiedenen Gruppen auf die Regionen Deutschlands verteilen. Zu beachten ist, dass die Schülerbefragung 2007/2008 keine Repräsentativität für einzelne Bundesländer beansprucht, weshalb nur Bundeslandkategorien unterschieden werden. $\mathrm{Zu}$ „Norddeutschland“ werden die Bundesländer Schleswig-Holstein, Hamburg und Niedersachsen zusammengefasst; zu „Westdeutschland“ die Bundesländer Nordrhein-Westfalen, Hessen, Rheinland-Pfalz und Saarland, zu „Süddeutschland“ Bayern und Baden-Württemberg, zu „Ostdeutschland" die fünf ostdeutschen Bundesländer. Berlin wurde bei der Einteilung nicht berücksichtigt.

Der Vergleich der Regionen führt zu zwei zentralen Befunden: Erstens zeigt sich, dass im Norden und Westen deutlich mehr Ab- als Aufsteiger zu finden sind als im Süden und im Osten, wo die Aufsteiger dominieren. Damit werden die Befunde des Berichts der Bertelsmann-Stiftung bestä- 
tigt (Bellenberg, 2012). Zweitens ergibt sich für den Osten Deutschlands noch eine Besonderheit: Der Anteil sonstiger Wechsler fällt hier sehr viel höher aus als in den anderen Regionen. Eine mögliche Erklärung könnte sein, dass die Schüler hier aufgrund der demographischen Veränderungen häufiger von Schulschließungen oder -zusammenlegungen betroffen sind, die einen horizontalen Schulwechsel nötig machen.

Der höchste Anteil an Schulwechslern ist in den Förderschulen festzustellen; drei Viertel der Schüler haben schon einmal einen Abstieg oder einen sonstigen Wechsel erlebt. Überraschend ist erneut der hohe Anteil an sonstigen Wechslern. Möglicherweise ist die Schulkarriere von Förderschülern stärker von Wechseln zwischen Förderschulen gekennzeichnet, weil sie aufgrund bspw. von Verhaltensproblemen an eine andere Schule verwiesen werden; möglicherweise erleben Förderschüler aber auch wiederholt Auf- und Abstiege, was ebenfalls zur Klassifikation in der sonstigen Gruppe führen würde. Die wenigsten Wechsel sind für Gymnasiasten festzustellen. Immerhin 8.9\% der Gymnasiasten sind Aufsteiger.

Für den Vergleich u. a. zwischen den Bundesländern ist es problematisch, dass die Schulstruktur und damit der Anteil integrierter Schulformen (Integrierte Haupt- und Realschule ${ }^{1}$ bzw. Gesamtschule) variiert. Aus diesem Grund findet sich in Abbildung 1 noch eine weitere Differenzierung nach dem angestrebten Schulabschluss. In integrierten Schulformen wurden die Jugendlichen gebeten, anzugeben, welchen Schulabschluss sie voraussichtlich an der besuchten Schule ablegen werden. Darüber lässt sich die Schulzweigzugehörigkeit bestimmen. Schüler an Schulen, die nur einen Abschluss anbieten, wird dieser als angestrebter Abschluss zugewiesen. Die gesamte Stichprobe betrachtet werden 31.5\% der Befragten einen Förder-/Hauptschulabschluss, 34.7\% einen Realschulabschluss und 33.8\% ein Abitur ablegen. Bezüglich der Wechsel zeigt sich, dass Schüler mit angestrebten Förder-/Hauptschulabschluss zu 19.3\% einen Abstieg und zu $5.1 \%$ einen Aufstieg erlebt haben. Bei Schülern mit angestrebtem Abitur liegt die Aufsteigerquote bei $8.4 \%{ }^{2}$

1 Abgekürzt: IHR. In den verschiedenen Bundesländern wird diese Schulform unterschiedlich bezeichnet, z. B. als Mittelschule, Sekundarschule usw.

2 Für die Auswertungen zu Schülern, die ein Abitur anstreben, mussten weitere 106 Personen als Personen mit fehlenden Werten eingestuft werden, da diese in Gesamtschulen unterrichtet wurden und gleichzeitig „Abitur" sowie „Abstieg“" angegeben haben, was nicht möglich ist. 


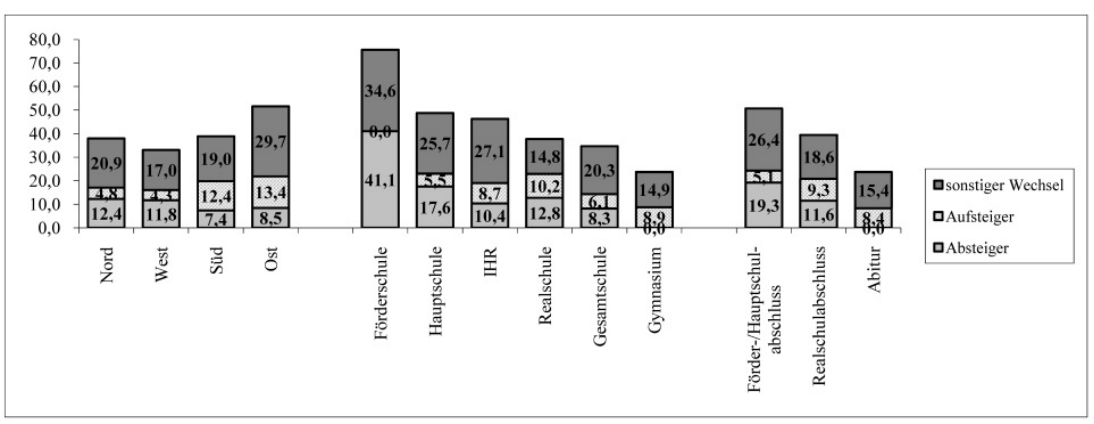

Abbildung 1. Schulwechsel nach Region und Schulform bzw. angestrebten Abschluss (in \%).

Die Auswertungen für die Regionen haben gezeigt, dass im Norden seltener auf- und häufiger abgestiegen wird als im Süden. Dieser Befund ist zurückhaltend zu interpretieren; d. h. er darf per se nicht dahingehend gedeutet werden, dass eine höhere Aufstiegsrate im Süden auf ein durchlässigeres oder gerechteres Schulsystem schließen lassen würde. Tabelle 2 verdeutlicht dies anhand der Verteilung der Schüler über angestrebte Schulabschlüsse, kombiniert mit der Anzahl an Auf- und Absteigern; auf die Darstellung Ostdeutschlands wird an dieser Stelle verzichtet.

Wird zuerst die Anzahl an Schülern, die ein Abitur (an Gymnasien oder Gesamtschulen) anstreben, betrachtet, so ergibt sich pro 1,000 Schüler im Norden eine Anzahl von 360 Abiturienten, von denen nur 24 Aufsteiger sind. Im Süden gibt es hingegen 1.6mal so viele Aufsteiger (39), die Gesamtzahl an Schülern, die ein Abitur ablegen werden, bleibt dennoch um mehr als ein Zehntel hinter der Anzahl des Nordens zurück (309 Abiturienten). Der höhere Aufsteigeranteil im Süden besagt also nur, dass hier zunächst mehr Schüler nicht ihren Leistungen entsprechend auf den Realschulzweig geschickt werden. Im Norden erhalten hingegen mehr Schüler von Beginn an die Möglichkeit, ein Abitur abzulegen. Dies geht gleichzeitig mit einem erhöhten Abstiegsrisiko einher: In Norddeutschland sind 52 Schüler von 1,000 Schülern in den Realschulzweig abgestiegen; in Süddeutschland waren es hingegen nur 31.

Zugleich ist im Norden auch das Abstiegsrisiko in den Förder/Hauptschulzweig höher, weil mehr Schüler den Realschulzweig besuchen können. Generell ist die Anzahl an Schülern im Förder/Hauptschulzweig im Norden aber nur etwa halb so hoch wie im Süden (248 vs. 398 Förder/Hauptschüler). Die Aufstiegsraten in den Realschulzweig sind im Norden dann auch entsprechend deutlich niedriger als im Süden (17 Aufsteiger vs. 
59 Aufsteiger), weil die Schüler von Beginn an häufiger diesem Zweig zugewiesen werden (im Norden insgesamt 392 Schüler, im Süden nur 293 Schüler).

Tabelle 2. Verteilung von 1,000 Schülern auf verschiedene angestrebte Abschlüsse nach Region.

\begin{tabular}{|c|c|c|c|}
\hline & Nord & West & Süd \\
\hline Förder-/Hauptschulabschluss: kein Wechsel & 104 & 151 & 215 \\
\hline Förder-/Hauptschulabschluss: Absteiger & 69 & 70 & 43 \\
\hline Förder-/Hauptschulabschluss: Aufsteiger & 7 & 12 & 27 \\
\hline Förder-/Hauptschulabschluss: sonstiger Wechsel & 68 & 74 & 113 \\
\hline Realschulabschluss: kein Wechsel & 244 & 235 & 166 \\
\hline Realschulabschluss: Absteiger & 52 & 45 & 31 \\
\hline Realschulabschluss: Aufsteiger & 17 & 17 & 59 \\
\hline Realschulabschluss: sonstiger Wechsel & 79 & 51 & 37 \\
\hline Abitur: kein Wechsel & 274 & 286 & 231 \\
\hline Abitur: Aufsteiger & 24 & 15 & 39 \\
\hline Abitur: sonstiger Wechsel & 61 & 45 & 39 \\
\hline
\end{tabular}

Die Befunde belegen, dass die Raten an Auf- und Absteigern nicht ohne die Verteilung der Schüler auf die Schulzweige betrachtet werden sollten, wenn sie als Indikator der Bildungschancen im Bundeslandvergleich genutzt werden. Dass im Norden letztlich die Bildungschancen (operationalisiert über den Anteil an Jugendlichen, die ein Abitur ablegen werden) besser sind als im Süden, dürfte im Wesentlichen darauf zurückzuführen sein, dass zumindest zum Zeitpunkt der Befragung die Schullaufbahnempfehlungen im Norden nur Orientierungen für die Eltern darstellten, im Süden hingegen bindend waren. Sich über die Empfehlung hinwegzusetzen, war im Süden entsprechend deutlich schwerer, was es notwendig macht, den dem Leistungsniveau angemessenen Zweig über Aufstiegsprozesse zu erreichen.

Die Untersuchung von Schülern, die auf- und absteigen, ist trotz dieser Befunde nicht obsolet. Zwar steht entsprechend den Auswertungen die Wahrscheinlichkeit des Auf-/Abstiegs mit schulstrukturellen Variablen, und hier insbesondere der Vergabe bzw. der Verbindlichkeit von Schullaufbahnempfehlungen in Zusammenhang. Weitere individuelle Merkmale könnten aber ebenso von Bedeutung sein. In Tabelle 3 werden zunächst 
verschiedene demographische Merkmale nach der Befragtengruppe unterschieden. ${ }^{3}$

Tabelle 3. Demographische Merkmale der Ab- und Aufsteiger (in \%).

\begin{tabular}{|c|c|c|c|c|}
\hline & $\begin{array}{l}\text { Anteil } \\
\text { männlich }\end{array}$ & $\begin{array}{c}\text { Anteil } \\
\text { Migrant }\end{array}$ & $\begin{array}{c}\text { Anteil Klas- } \\
\text { se wieder- } \\
\text { holt }\end{array}$ & $\begin{array}{l}\text { Anteil Klas- } \\
\text { se wieder- } \\
\text { holt (nur } \\
\text { männlich) }\end{array}$ \\
\hline Gesamt & 51.3 & 27.4 & 24.0 & 26.7 \\
\hline kein Wechsel & 49.2 & 23.9 & 15.8 & 18.2 \\
\hline Absteiger & 59.2 & 30.8 & 47.1 & 48.4 \\
\hline Aufsteiger & 56.5 & 26.0 & 24.7 & 25.8 \\
\hline $\begin{array}{l}\text { Förder-/Hauptschulabschluss: } \\
\text { kein Wechsel }\end{array}$ & 55.0 & 37.1 & 28.5 & 29.6 \\
\hline $\begin{array}{l}\text { Förder-/Hauptschulabschluss: } \\
\text { Absteiger }\end{array}$ & 60.6 & 37.2 & 51.9 & 51.4 \\
\hline $\begin{array}{l}\text { Förder-/Hauptschulabschluss: } \\
\text { Aufsteiger }\end{array}$ & 63.2 & 33.7 & 33.1 & 35.3 \\
\hline Realschulabschluss: kein Wechsel & 49.5 & 21.9 & 17.4 & 19.4 \\
\hline Realschulabschluss: Absteiger & 57.1 & 22.0 & 40.6 & 44.0 \\
\hline Realschulabschluss: Aufsteiger & 54.8 & 24.3 & 26.2 & 26.6 \\
\hline Abitur: kein Wechsel & 45.5 & 18.1 & 7.2 & 9.2 \\
\hline Abitur: Aufsteiger & 54.1 & 23.9 & 18.3 & 18.5 \\
\hline
\end{tabular}

Auffällig ist, dass sowohl unter den Auf- als auch unter den Absteigern der Anteil männlicher Befragter erhöht ist: 59.2\% der Absteiger, aber auch $56.5 \%$ der Absteiger sind männlich, entsprechend nur 40.8 bzw. 43.5\% weiblich. Schüler ohne Schulwechsel sind aber nur zu 49.2\% männlich. Der höhere Anteil männlicher Auf- bzw. Absteiger zeigt sich in Bezug auf alle Schulzweige. In den Schulwechselquoten zeigt sich damit eine Dimension der 'Krise der Jungen': Sie werden häufiger einem geringeren Schulzweig zugewiesen, welchen sie dann über den Aufstieg verlassen; oder sie werden einem zu hohem Zweig zugewiesen, kommen mit den Anforderungen hier häufiger nicht zurecht und steigen $a b$. Von allen

3 Hier und im Folgenden wird aus Gründen der Übersichtlichkeit darauf verzichtet, die Gruppe der sonstigen Wechsler mit aufzuführen. 
männlichen Jugendlichen haben $11.6 \%$ einen Abstieg erlebt, von allen weiblichen Jugendlichen nur 8.2\%, also etwa ein Drittel weniger.

Auch der Migrantenanteil ist unter den Auf-, insbesondere aber unter den Absteigern erhöht ${ }^{4}$ : $30.8 \%$ der Absteiger sind Migranten aber nur 23.9\% der Schüler ohne Wechsel. Dieser Effekt kommt aber nur deshalb zustande, weil Migranten eher auf niedrigere Schulform absteigen. Werden bspw. nur die Schüler betrachtet, die einen Förder-/Hauptschulabschluss anstreben, so liegt der Migrantenanteil bei den Nichtwechslern mit 37.1\% genauso hoch wie der Anteil bei den Absteigern (37.2\%). Grundsätzlich $\mathrm{zu}$ fragen ist damit zwar, warum der Migrantenanteil in niedrigeren Schulformen erhöht ist, Migranten also in diesen Schulformen im Vergleich zu Deutschen überrepräsentiert sind; hinsichtlich des Schulwechsels (und hier insbesondere des Absteigens) kann aber nun nicht von einer zusätzlichen Benachteiligung gesprochen werden, da sonst die Anteile im Vergleich zu den Schülern ohne Wechsel erhöht sein sollten.

Abgebildet ist in Tabelle 3 zuletzt der Anteil an Jugendlichen, die eine Klasse wiederholt haben. Nicht überraschend ist, dass dieser Anteil unter den Absteiger mit 47.1\% deutlich höher ist als bei den Schülern ohne Wechsel. Zusätzlich gilt aber auch, dass die Aufsteiger häufiger sitzen geblieben sind. Möglicherweise ist dies kurz nach dem Aufstieg geschehen, so dass die Schüler anfänglich mit dem neuen Anspruchsniveau nicht zu Recht gekommen sind, sich zwischenzeitlich aber damit arrangiert haben. Jungen bleiben insgesamt häufiger sitzen als Mädchen, weshalb die Auswertungen zum Sitzenbleiben noch einmal getrennt für die männlichen Befragten abgebildet sind. Die Ergebnisse ändern sich hierdurch aber nicht.

\subsection{Schulbezogene Einstellungen und Erfahrungen}

Tabelle 4 stellt für die Ab- und Aufsteiger verschiedene schulbezogene Einstellungen und Erfahrungen gegenüber. Zunächst wird dabei die Mathematiknote betrachtet. Diese wurde hier ausgewählt, weil sie bspw. weitestgehend unabhängig von der ethnischen Herkunft eines Befragten ist.

4 Jugendliche wurden als deutsch klassifiziert, wenn sie in Deutschland geboren worden sind und die deutsche Staatsangehörigkeit besitzen und wenn dies ebenso auf die beiden leiblichen Eltern zutrifft. Sobald bei der Staatsangehörigkeit bzw. beim Geburtsland ein nichtdeutsches Land genannt wurde, zählt ein Jugendlicher als Schüler mit Migrationshintergrund. 
Erfasst wurde sie als Selbstauskunft mit der herkömmlichen Notenskala von 1 bis 6. Deutlich wird, dass sowohl Ab- als auch Aufsteiger durchschnittlich eine um ca. 0.1 Punkte schlechtere Mathematiknote aufweisen. Bei Schülern, die ein Abitur anstreben, beträgt der Notenunterschied sogar 0.2 Punkte. Dies dürfte ebenfalls darauf zurückzuführen sein, dass die Aufsteiger zwar in der Schulform, von der sie kommen, überdurchschnittliche Leistungen erzielt haben (sonst wäre kein Aufstieg möglich gewesen); das Leistungsniveau an der neuen Schule liegt aber höher, so dass sie hier nur unterdurchschnittliche Leistungen erzielen. Der höhere Schulabschluss wird also mit schlechteren Schulnoten erkauft. Fraglich bleibt dabei, ob die Schüler bessere Leistungen erzielen würden, wenn sie von Anfang an auf die höhere Schulform gegangen wären und nicht erst mit einer mehr oder weniger langen Verzögerung.

Bezüglich der Schulbindung, die über die zwei Aussagen „An meiner Schule gefällt es mir wirklich gut" und „Ich gehe gern zur Schule“ erfasst wurde (Antwortvorgaben: von „1 - stimmt nicht" bis , 4 - stimmt genau“), zeigt sich, dass sich Schüler ohne Wechsel und Aufsteiger nicht unterscheiden, auch dann nicht, wenn ein Vergleich innerhalb der einzelnen Schulzweige erfolgt. Eine etwas geringere Bindung ergibt sich dagegen für Absteiger.

Die geringere Bindung an die Schule zeigt sich noch stärker, wenn das Schulschwänzen betrachtet wird. Absteiger haben zu 38.0\% mindestens einen Tag die Schule geschwänzt, bei Schülern ohne Wechsel bzw. bei Aufsteigern liegt die Quote um mehr als zehn Prozentpunkte niedriger. Die höhere Schwänzbereitschaft findet sich sowohl bei Absteigern, die einen Förder-/Hauptschulabschluss anstreben, als auch bei Absteigern, die einen Realschulabschluss anstreben. Es lässt sich insofern folgern, dass bei Absteigern eine Distanzierung von der Schule einsetzt, die mit schlechteren Noten und stärkerer innerer oder äußerer Abkehr von der Schule einhergeht.

Werden die Raten an Schülern betrachtet, die Opfer physischer Übergriffe durch Mitschüler geworden sind, ergeben sich nur geringe Differenzen zwischen den Gruppen. Gefragt wurde danach, ob im letzten Schulhalbjahr mindestens einmal ein Übergriff des Schlagens/Tretens oder des Erpressens stattgefunden hat. Auffällig sind die Absteiger allerdings, wenn die Täterraten betrachtet werden: Absteiger treten deutlich häufiger als physische Gewalttäter in Erscheinung als Schüler ohne Wechsel oder Aufsteiger.

Die Aufsteiger sind hinsichtlich ihrer schlechteren Schulleistungen sowie hinsichtlich einer weiteren Variablen auffällig: Sie berichten etwas 
häufiger davon, Schulangst zu haben. Diese wurde über fünf Aussagen wie „Ich habe Angst vor Klassenarbeiten“ und „Ich kann oft nicht einschlafen, weil ich mir so viele Gedanken wegen der Schule machen muss" gemessen (Antwortvorgaben: von ,1 - stimmt nicht" bis „4 - stimmt genau“). Schulabsteiger stimmen diesen Aussagen seltener zu, was in Übereinstimmung mit ihrer geringen Schulbindung steht; sie kümmern sich weniger um Schulangelegenheiten und werden durch Tests o. ä. weniger gestresst. Aufsteiger hingegen, insbesondere Aufsteiger an Gymnasien, machen sich mehr Sorgen um ihre Schulleistungen und haben dann auch häufiger Angst.

Tabelle 4. Schulbezogene Einstellungen und Erfahrungen der Ab- und Aufsteiger (Mittelwerte bzw. in \%).

\begin{tabular}{|c|c|c|c|c|c|c|}
\hline & $\begin{array}{l}\text { Mathe- } \\
\text { matiknote }\end{array}$ & $\begin{array}{c}\text { Schul- } \\
\text { bindung }\end{array}$ & $\begin{array}{c}\text { mind. } \\
\text { einen Tag } \\
\text { ge- } \\
\text { schwänzt }\end{array}$ & $\begin{array}{l}\text { Opfer } \\
\text { Schul- } \\
\text { gewalt }\end{array}$ & $\begin{array}{l}\text { Täter } \\
\text { Schul- } \\
\text { gewalt }\end{array}$ & $\begin{array}{l}\text { Schul- } \\
\text { angst }\end{array}$ \\
\hline kein Wechsel & 3.09 & 2.70 & 23.4 & 20.2 & 21.9 & 1.97 \\
\hline Absteiger & 3.18 & 2.52 & 38.0 & 24.1 & 34.5 & 1.83 \\
\hline Aufsteiger & 3.21 & 2.68 & 25.9 & 22.4 & 24.4 & 2.03 \\
\hline $\begin{array}{l}\text { Förder-/Hauptschulabschluss: } \\
\text { kein Wechsel }\end{array}$ & 3.22 & 2.52 & 32.2 & 24.0 & 30.4 & 1.90 \\
\hline $\begin{array}{l}\text { Förder-/Hauptschulabschluss: } \\
\text { Absteiger }\end{array}$ & 3.12 & 2.46 & 42.3 & 25.1 & 38.0 & 1.77 \\
\hline $\begin{array}{l}\text { Förder-/Hauptschulabschluss: } \\
\text { Aufsteiger }\end{array}$ & 3.18 & 2.46 & 33.3 & 26.8 & 32.8 & 1.91 \\
\hline $\begin{array}{l}\text { Realschulabschluss: } \\
\text { kein Wechsel }\end{array}$ & 3.17 & 2.68 & 20.8 & 21.2 & 22.9 & 2.01 \\
\hline $\begin{array}{l}\text { Realschulabschluss: } \\
\text { Absteiger }\end{array}$ & 3.26 & 2.62 & 31.8 & 22.8 & 29.4 & 1.91 \\
\hline $\begin{array}{l}\text { Realschulabschluss: } \\
\text { Aufsteiger }\end{array}$ & 3.27 & 2.68 & 24.8 & 23.0 & 26.0 & 2.05 \\
\hline Abitur: kein Wechsel & 2.94 & 2.82 & 20.5 & 17.1 & 16.4 & 1.96 \\
\hline Abitur: Aufsteiger & 3.15 & 2.81 & 23.3 & 19.2 & 18.0 & 2.06 \\
\hline
\end{tabular}

\subsection{Familiärer Hintergrund}

Schulabsteiger kommen entsprechend den Ergebnissen aus Tabelle 5 überproportional häufig aus Familien, in denen Trennungen oder Scheidungen stattgefunden haben: $35.3 \%$ der Absteiger leben derzeit nicht mit beiden leiblichen Elternteilen zusammen, bei den Schülern ohne Wechsel 
sind es nur 25.1\%; ein entsprechender Unterschied findet sich bei Jungen wie bei Mädchen (nicht dargestellt). Auch für die Aufsteiger gilt, dass sie häufiger aus Elternhäusern mit Trennungserfahrungen stammen; die Unterschiede fallen aber geringer aus. Inwieweit die mit Trennungen und Scheidungen verbundenen, innerfamiliären Belastungen ein Auslöser für schlechte Schulleistungen und damit letztlich auch Abstiege sind, kann mit den vorhandenen Daten nicht beantwortet werden. Hierzu wären Längsschnittstudien notwendig. Es ist aber durchaus nahe liegend, dass innerfamiliärer Stress eine Ursache für Schulabstiege ist, insofern in der Vergangenheit in Bezug auf verschiedene Verhaltensweisen belegt wurde, dass Trennungen/Scheidungen Problemverhaltensweisen wahrscheinlicher machen (vgl. z. B. Farrington, 2010; Walper, 2009).

Für die Absteiger ergibt sich zugleich, dass sie häufiger aus Familien kommen, die von staatlichen Transferleistungen abhängig sind. Damit sind Familien gemeint, bei denen mindestens ein Elternteil derzeit arbeitslos ist oder die Sozialhilfe/Arbeitslosengeld II beziehen. Allerdings gilt dies nur in Bezug auf Schüler, die einen Förder-/Hauptschulabschluss anstreben: Während $21.9 \%$ der Absteiger aus von Transferleistungen abhängigen Familien kommen, sind dies unter Schülern ohne Wechsel nur $17.5 \%$. Bei Absteigern in den mittleren Schulsektor ist die höhere Transferleistungsquote nicht feststellbar. Es zeigt sich dagegen, dass die Aufsteiger häufiger aus Familien mit Transferleistungsbezug stammen; bei den Schülern, die ein Abitur anstreben, ist dies besonders deutlich (ohne Wechsel: $6.2 \%$, Aufsteiger: 12.2\%). Werden zusätzlich die Befunde zum Bildungsniveau im Elternhaus einbezogen, lässt sich folgende These aufstellen: Der Aufstieg wird häufiger von Schülern bewältigt, die aus unteren Sozialschichten stammen und die auch aufgrund dieser Herkunft zunächst nicht für die höhere Schullaufbahn empfohlen wurden. Ihre Leistungen hätten die Zuweisung auf eine höhere Schule aber möglicherweise gerechtfertigt. Angesprochen ist damit die zentrale Rolle der Lehrkräfte, die Laufbahnempfehlungen verteilen. Bei dieser Entscheidung stützen sie sich offenbar nicht allein auf die Leistungen der Schüler, sondern es werden auch andere Kriterien herangezogen, bspw. Ansichten darüber, ob ein Schüler im Elternhaus genügend Unterstützung erfährt, um den Anforderungen in höheren Schulformen zu entsprechen. Bei Schülern aus sozial schwächerem Elternhaus wird die Entscheidung bei gleichem Notendurchschnitt wie bei Schülern aus stärkerem Elternhaus eventuell häufiger negativ in Bezug auf die Empfehlung auf eine höhere Schulform ausfallen.

In Bezug auf den Bildungsabschluss im Elternhaus ergeben sich im Einzelnen folgende Befunde: Keinen oder einen einfachen Schulabschluss 
(höchstens Hauptschule) weisen 24.2\% der Ab- und 21.9\% der Aufsteiger auf, einen höheren Abschluss (mindestens Fachabitur) 33.5\% der Ab- und $35.6 \%$ der Aufsteiger. Wenn wir uns auf die Schüler konzentrieren, die ein Abitur anstreben, so zeigt sich, dass die Aufsteiger deutlich seltener aus Elternhäusern mit hohem Bildungsabschluss stammen (50.3\% zu 60.1\%). Die Absteiger der anderen Abschlussgruppen kommen hingegen aus höher gebildeten Elternhäusern. Im Umkehrschluss zur oben geäußerten These gilt für diese Schüler, dass sie aufgrund des höheren Bildungsniveaus im Elternhaus wahrscheinlich häufiger zum Besuch höherer Schulformen empfohlen wurden, wobei die Leistungen diesen Empfehlungen nicht entsprachen. Sie werden dann durch den Abstieg ihrem eigentlichen Leistungsniveau zugewiesen.

Tabelle 5. Familiärer Hintergrund der Ab- und Aufsteiger (in \%).

\begin{tabular}{|c|c|c|c|c|c|}
\hline & $\begin{array}{c}\text { nicht mit } \\
\text { beiden leibli- } \\
\text { chen Eltern } \\
\text { zusammen } \\
\text { lebend }\end{array}$ & $\begin{array}{c}\text { abhängig von } \\
\text { staatlichen } \\
\text { Transfer- } \\
\text { leistungen }\end{array}$ & $\begin{array}{c}\text { kein Ab- } \\
\text { schluss/ einfa- } \\
\text { cher Ab- } \\
\text { schluss }\end{array}$ & $\begin{array}{l}\text { höherer Ab- } \\
\text { schluss }\end{array}$ & $\begin{array}{l}\text { schwere elter- } \\
\text { liche Gewalt } \\
\text { in Kindheit }\end{array}$ \\
\hline kein Wechsel & 25.1 & 10.3 & 19.9 & 39.8 & 12.7 \\
\hline Absteiger & 35.3 & 16.9 & 24.2 & 33.5 & 19.0 \\
\hline Aufsteiger & 27.6 & 13.9 & 21.9 & 35.6 & 15.7 \\
\hline $\begin{array}{l}\text { Förder-/Hauptschulabschluss: } \\
\text { kein Wechsel }\end{array}$ & 30.1 & 17.5 & 40.3 & 17.0 & 17.1 \\
\hline $\begin{array}{l}\text { Förder-/Hauptschulabschluss: } \\
\text { Absteiger }\end{array}$ & 38.7 & 21.9 & 31.4 & 25.5 & 22.0 \\
\hline $\begin{array}{l}\text { Förder-/Hauptschulabschluss: } \\
\text { Aufsteiger }\end{array}$ & 30.4 & 16.5 & 35.9 & 23.6 & 19.5 \\
\hline $\begin{array}{l}\text { Realschulabschluss: kein } \\
\text { Wechsel }\end{array}$ & 25.5 & 10.2 & 20.9 & 30.4 & 13.4 \\
\hline Realschulabschluss: Absteiger & 30.6 & 9.8 & 14.3 & 44.2 & 14.9 \\
\hline Realschulabschluss: Aufsteiger & 28.5 & 14.1 & 24.5 & 28.7 & 14.5 \\
\hline Abitur: kein Wechsel & 21.8 & 6.2 & 7.9 & 60.1 & 9.7 \\
\hline Abitur: Aufsteiger & 25.0 & 12.2 & 11.6 & 50.3 & 14.8 \\
\hline
\end{tabular}

Die Zahlen aus Tabelle 5 belegen, dass die Schüler, die niedrigere Schulabschlüsse anstreben, seltener aus Elternhäusern mit hoher Bildung kommen. Dies wird noch einmal in Abbildung 2 verdeutlicht. Für Gesamtdeutschland gilt, dass Schüler aus einem niedrig gebildetem Elternhaus nur zu 13.0\% ein Abitur anstreben, dafür zu 54.4\% einen Förder- oder Hauptschulabschluss. Schüler aus hoch gebildeten Elternhäusern streben 
hingegen zu über der Hälfte ein Abitur an (53.9\%) und nur zu 16.0\% einen Förder- oder Hauptschulabschluss. Diese Zusammenhänge zwischen der elterlichen Bildung und dem angestrebten Schulabschluss ergeben sich für alle Regionen, d. h. dies ist im Norden nicht anders als im Süden. Aufgrund des anteilsmäßig größeren Hauptschulsektors im Süden liegen die Anteile an Schülern, die einen Förder-/Hauptschulabschluss anstreben, hier für alle elterlichen Bildungsniveaus höher. Interessant ist, dass der Anteil an Schülern aus höher gebildeten Elternhäusern, die ein Abitur anstreben, im Süden ähnlich hoch ausfällt wie im Norden. Es gelingt der höher gebildeten Elternschaft also trotz anderer Schulstruktur ebenso oft, ihren Kindern gymnasiale Bildung zukommen zu lassen.

Jugendliche aus mittel gebildeten Elternhäusern streben in Norddeutschland zu 27.3\% ein Abitur an, in Süddeutschland zu 25.3\%. Hierin unterscheiden sich die Gebiete also kaum voneinander. Allerdings zeigt sich dann, wenn danach gefragt wird, ob diese Schüler von Beginn an den entsprechenden Bildungszweig besuchten oder erst über Aufstiegsprozesse hierher gelangt sind, ein beachtlicher Unterschied: In Norddeutschland strebt jeder 15. dieser Schüler aufgrund eines Aufstiegs ein Abitur an (7.0\%), in Süddeutschland hingegen jeder sechste Schüler (15.6\%). Vergleichbares gilt in Bezug auf Schüler aus niedrig gebildeten Elternhäusern, die ein Abitur anstreben: In Norddeutschland ist der Anteil ähnlich hoch wie in Süddeutschland (13.0 bzw. 11.1\%). In Norddeutschland waren aber deutlich mehr Schüler als in Süddeutschland von Anfang an in dieser Schulform. Der Anteil an Aufsteigern liegt in Norddeutschland bei 8.8\%, in Süddeutschland hingegen bei 23.4\%. Dass es in Süddeutschland mehr Aufstiege als in Norddeutschland gibt, ist daher z. T. damit zu begründen, dass hier Schüler aus niedrig oder mittel gebildeten Elternhäusern im Übergang auf das Gymnasium zunächst benachteiligt und auf eine niedrigere Schulform geschickt wurden. Diese Benachteiligung wurde durch Aufstiegsmobilität überwunden, so dass im Endeffekt die Verteilung über die Abschlüsse in Nord- und Süddeutschland recht ähnlich aussieht; in Süddeutschland mussten die Schüler aber mehr Anstrengungen unternehmen als in Norddeutschland, wo von Anfang an eine Zuteilung zum angemessenen Schulniveau vorgenommen wurde. 


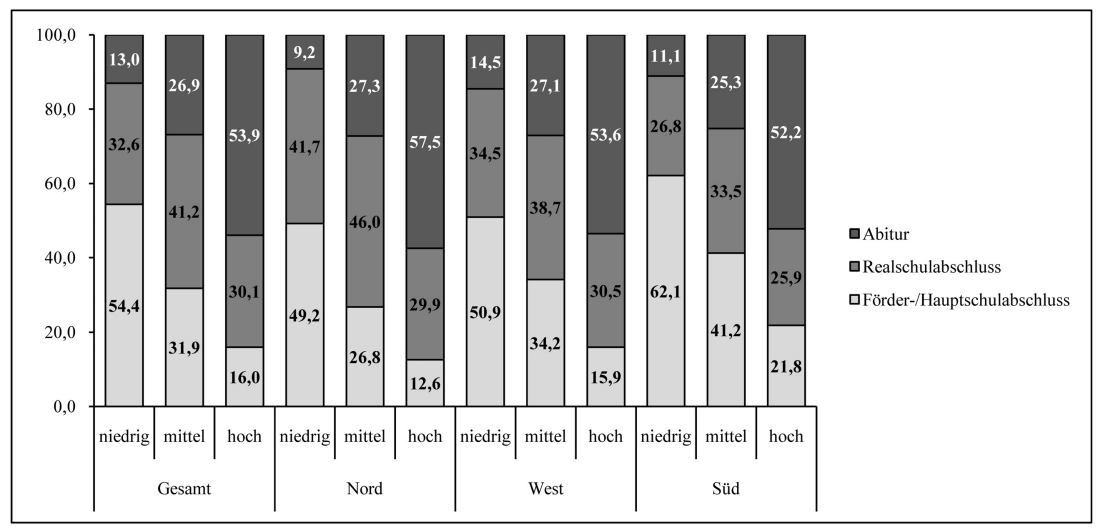

Abbildung 2. Angestrebter Schulabschluss nach elterlichem Bildungsniveau und Region (in \%).

In Tabelle 5 ist hinsichtlich des Elternhauses noch eine weitere Variable dargestellt, die die Erziehungskultur und nicht den sozio-ökonomischen Status beschreibt. Die Aufsteiger, insbesondere aber die Absteiger, berichten häufiger davon, schwere elterliche Gewalt in der Kindheit erfahren zu haben. Angegeben werden sollte, ob man mit einem Gegenstand geschlagen, mit der Faust geschlagen oder getreten bzw. geprügelt oder zusammengeschlagen wurde. Absteiger bestätigen dies zu 19.0\%, Befragte ohne Wechsel zu 12.7\%. Ähnlich wie bereits bei den Trennungserlebnissen ausgeführt, dürfte das erhöhte Stressniveau in Familien mit Gewaltanwendung in der Erziehung eine Erklärung für diesen Befund darstellen. Dass auch Aufsteiger häufiger von Gewalterfahrungen berichten, ist möglicherweise damit zu begründen, dass es sich hier häufiger um Schüler aus sozial schwächeren Sozialschichten handelt, in denen die Gewaltanwendung verbreiteter ist (s. u.).

\subsection{Persönlichkeit}

Hinsichtlich der Persönlichkeit sollen nachfolgend zwei Komplexe betrachtet werden: Einerseits diagnostizierte Probleme und zweitens verschiedene Einschätzungen und Verhaltensweisen, die durchaus eine Folge von Ab- und Aufstiegsprozessen darstellen können. In Tabelle 6 ist zunächst dargestellt, welcher Prozentsatz der Schüler angab, dass schon einmal ein Arzt oder Psychologe eine Aufmerksamkeitsstörung festgestellt 
hat. Absteiger bestätigen dies doppelt so häufig wie Schüler ohne Wechsel (11.5 zu 5.8\%). Erneut kann über die Ursache-Wirkungs-Beziehung gestritten werden; eine Klärung dieser Frage ist mittels der vorliegenden Querschnittsbefragung nicht möglich. Wenn aber davon ausgegangen werden kann, dass einer ADHS-Diagnose ein Krankheitsbild zugrunde liegt, welches i. d. R. bereits recht früh in der Kindheit ausgebildet wird und damit genetisch bedingt sein kann, dürfte gelten, dass die ADHSSymptomatik eher die Ursache des Abstiegs ist als die Folge. Die Schüler entsprechen häufiger nicht den schulischen Anforderungen hinsichtlich eines angemessenen Verhaltens im Unterricht. Darunter leiden auch die Schulleistungen, was dann letztendlich zu einer Versetzung auf ein niedriges Schulniveau führt. Dass auch unter den Aufsteigern ein erhöhter ADHS-Anteil zu verzeichnen ist, könnte dann damit in Zusammenhang stehen, dass bei Schülern, die aufgrund ihrer ADHS-Diagnose zunächst für eine niedrigere Schulform empfohlen wurden, früher oder später erkannt wird, dass sie leistungsfähiger sind. Dementsprechend wird ihnen dann nachträglich die Chance eingeräumt, eine dem tatsächlichen Leistungsniveau entsprechende Schulform zu besuchen.

Aufgeführt in Tabelle 6 sind zusätzlich die Werte für männliche und weibliche Befragte. Aus diesen geht erstens hervor, dass weibliche Jugendliche deutlich seltener eine ADHS-Diagnose berichten. Zweitens gilt aber für Jungen wie für Mädchen, dass Absteiger etwa doppelt so häufig eine ADHS-Diagnose berichten wie Schüler ohne Schulwechsel. Insofern ist ADHS für beide Geschlechter ein Risikomerkmal.

Hinsichtlich zwei weiterer Auffälligkeiten ergeben sich im Wesentlichen nur Höherbelastungen für die Absteiger. Gefragt wurden die Schüler, ob sie schon einmal wegen einer Angsterkrankung oder einer Depression in Behandlung waren. Bei Absteigern liegen die Raten bei beiden Erkrankungen mindestens 1.5-mal so hoch im Vergleich zu den Schülern ohne Wechsel. Die Erkrankungen können einerseits die Ursache des Abstiegs darstellen, weil die Schüler sich weniger auf ihre schulische Arbeit konzentrieren können. Gleichwohl können Ängste und Depressionen auch aus Abstiegen resultieren, die Selbstzweifel bzgl. der eigenen Leistungsfähigkeit entstehen lassen. Hinsichtlich der Angstbehandlung gilt, dass nur für weibliche Absteiger Auffälligkeiten bestehen, hinsichtlich der Depressionsbehandlung hingegen wiederum für beide Geschlechter. Bei beiden Auffälligkeiten sind Mädchen höher belastet als Jungen. 
Tabelle 6. Psychische Auffälligkeiten der Ab- und Aufsteiger (in \%; in Klammern: Werte für männliche/weibliche Jugendliche).

\begin{tabular}{|c|c|c|c|}
\hline & $\begin{array}{c}\text { ADHS- } \\
\text { Diagnose }\end{array}$ & $\begin{array}{c}\text { Behandlung } \\
\text { Angst }\end{array}$ & $\begin{array}{c}\text { Behandlung De- } \\
\text { pression }\end{array}$ \\
\hline kein Wechsel & $5.8(7.7 / 4.0)$ & $4.3(2.8 / 5.8)$ & $4.0(2.9 / 5.0)$ \\
\hline Absteiger & $11.5(13.9 / 8.1)$ & $6.3(2.8 / 11.2)$ & $7.7(5.5 / 10.8)$ \\
\hline Aufsteiger & $7.4(9.0 / 5.4)$ & $4.6(3.1 / 6.5)$ & $4.2(2.7 / 6.1)$ \\
\hline $\begin{array}{l}\text { Förder-/Hauptschulabschluss: } \\
\text { kein Wechsel }\end{array}$ & 9.0 & 5.0 & 4.4 \\
\hline $\begin{array}{l}\text { Förder-/Hauptschulabschluss: } \\
\text { Absteiger }\end{array}$ & 12.2 & 5.8 & 7.4 \\
\hline $\begin{array}{l}\text { Förder-/Hauptschulabschluss: } \\
\text { Aufsteiger }\end{array}$ & 10.1 & 3.3 & 2.9 \\
\hline $\begin{array}{l}\text { Realschulabschluss: kein Wech- } \\
\text { sel }\end{array}$ & 6.1 & 4.2 & 3.8 \\
\hline Realschulabschluss: Absteiger & 10.3 & 6.7 & 8.1 \\
\hline Realschulabschluss: Aufsteiger & 7.7 & 4.7 & 4.9 \\
\hline Abitur: kein Wechsel & 3.7 & 4.0 & 3.9 \\
\hline Abitur: Aufsteiger & 5.5 & 5.0 & 3.7 \\
\hline
\end{tabular}

Die Absteiger sind zudem in verschiedenen Verhaltensbereichen auffällig, die in einem Zusammenhang mit der Bewältigung von Schulwechselerfahrungen gebracht werden können (vgl. Tabelle 7). Hinsichtlich verschiedener, die psychische Konstitution betreffenden Einschätzungen sind die Unterschiede zu den Schülern ohne Wechselerfahrungen eher gering. Die Lebenszufriedenheit, die über die Aussage „Wie zufrieden bist du mit deinem Leben insgesamt“ (,1 - überhaupt nicht zufrieden“ bis „,4-sehr zufrieden") erfasst wurde, wird sowohl von den Ab- als auch den Aufsteigern etwas geringer eingeschätzt; bei den Mädchen fallen die Differenzen etwas größer aus. Die Aufsteiger in den Abiturzweig sowie die Absteiger in den Förder-/Hauptschulzweig weisen die niedrigste Lebenszufriedenheit auf. Beim emotionalen Unwohlbefinden sind die Aufsteiger etwas auffälliger als die anderen Gruppen; sie schätzen ihr Wohlbefinden schlechter ein. Bewertet werden sollten hier vier Aussagen wie „In der letzten Woche habe ich mich allein gefühlt" oder "In der letzten Woche habe ich mich ängstlich und unsicher gefühlt" (,1 - nie" bis ,,5-immer"). Bei den Mädchen gibt es stärkere Unterschiede zwischen den NichtWechslern einerseits und den Auf- bzw. Absteigern anderseits.

Erfragt wurde zusätzlich, ob die Schüler zumindest selten Selbstmordgedanken haben. Die Auf- wie die Absteiger berichten etwas häufiger 
hiervon, wobei die Unterschiede eher gering ausfallen. Mädchen haben daneben deutlich häufiger als Jungen Selbstmordgedanken; bezüglich der Gruppenunterschiede ergeben sich aber vergleichbare Tendenzen.

Hinsichtlich der Verhaltensweisen sind die Unterschiede deutlicher ausgeprägt. Einen Selbstmordversuch haben 13.2\% der Absteiger ausgeführt, aber nur etwa halb so viele Schüler ohne Wechsel (7.1\%). Insbesondere für die Aufsteiger in den Abiturzweig ergibt sich eine im Vergleich zu den Nicht-Wechslern höhere Selbstmordversuchsrate. Im Vergleich von Jungen und Mädchen weisen die Absteiger beide Male erhöhte Selbstmordversuchsraten auf; Mädchen berichten zugleich deutlich häufiger von solchen Versuchen.

Rauschtrinken, erfasst über die Frage, ob man im zurückliegenden Monat zu mindestens einer Trinkgelegenheit fünf oder mehr alkoholische Getränke nacheinander zu sich genommen hat, wird besonders häufig von den Absteigern praktiziert - von Jungen wie von Mädchen. Die Aufsteiger berichten ein solches Verhalten vergleichbar häufig wie die Schüler ohne Wechsel, auch innerhalb der unterschiedenen Bildungszweige. Der Cannabiskonsum ist ebenfalls unter den Absteigern häufiger verbreitet; dargestellt ist der Anteil an Schülern, die mindestens monatlich Cannabis konsumieren. Bei den Absteigern liegt dieser Anteil fast dreimal so hoch wie bei den Aufsteigern und bei den Schülern ohne Wechsel. Sowohl für das Rauschtrinken als auch den Cannabiskonsum gilt, dass Jungen häufiger hiervon berichten. Die besondere Auffälligkeit der Absteiger zeigt sich aber bei Jungen wie bei Mädchen.

Letztlich deutet sich damit an, dass die Absteiger möglicherweise im Vorfeld des Abstiegs, denkbar aber auch als Folge davon, verhaltensbezogene Auffälligkeiten zeigen, die sich z. T. bereits als psychische Erkrankungen diagnostizieren lassen, z. T. aber auch jenseits davon bspw. im Bereich des Alkohol- und Cannabiskonsums zu Tage treten. Die Absteiger sind damit eher externalisierend auffällig. Die Aufsteiger wiederum, und hier vor allem die Aufsteiger in den Abiturzweig, berichteten stärker von psychischen Belastungen, die sich weniger stark externalisieren. Die Lebenszufriedenheit ist geringer, Selbstmordgedanken und -versuche finden sich in dieser Gruppe häufiger. Die Aufsteiger, deren Aufstieg als Erfolgsund Selbstwirksamkeitserlebnis eingestuft werden könnte, scheinen dies nicht notwendigerweise so wahr zu nehmen. Sie fühlen sich demgegenüber häufiger nicht wohl in ihrer neuen Situation. 
Tabelle 7. Mögliche Folgen des Ab- und Aufstiegs (Mittelwerte bzw. in $\%$; in Klammern: Werte für männliche/weibliche Jugendliche).

\begin{tabular}{|c|c|c|c|c|c|c|}
\hline & $\begin{array}{l}\text { Lebenszu- } \\
\text { friedenheit }\end{array}$ & $\begin{array}{c}\text { emotiona- } \\
\text { les } \\
\text { Unwohl- } \\
\text { befinden } \\
\end{array}$ & $\begin{array}{l}\text { Selbst- } \\
\text { mord- } \\
\text { gedanken }\end{array}$ & $\begin{array}{l}\text { Selbst- } \\
\text { mord- } \\
\text { versuch }\end{array}$ & $\begin{array}{l}\text { Rausch- } \\
\text { trinken }\end{array}$ & $\begin{array}{l}\text { Cannabis- } \\
\text { konsum }\end{array}$ \\
\hline kein Wechsel & $\begin{array}{c}3.22 \\
(3.32 / 3.13)\end{array}$ & $\begin{array}{c}2.05 \\
(1.99 / 2.11)\end{array}$ & $\begin{array}{c}37.5 \\
(27.2 / 47.4)\end{array}$ & $\begin{array}{c}7.1 \\
(3.9 / 10.2)\end{array}$ & $\begin{array}{c}52.1 \\
(57.5 / 46.9)\end{array}$ & $\begin{array}{c}2.8 \\
(4.0 / 1.6)\end{array}$ \\
\hline Absteiger & $\begin{array}{c}3.15 \\
(3.26 / 2.99)\end{array}$ & $\begin{array}{c}2.07 \\
(1.97 / 2.21)\end{array}$ & $\begin{array}{c}41.3 \\
(31.6 / 55.6)\end{array}$ & $\begin{array}{c}13.2 \\
(7.4 / 21.6)\end{array}$ & $\begin{array}{c}63.8 \\
(66.9 / 59.2)\end{array}$ & $\begin{array}{c}8.8 \\
(10.5 / 6.3)\end{array}$ \\
\hline Aufsteiger & $\begin{array}{c}3.16 \\
(3.25 / 3.06)\end{array}$ & $\begin{array}{c}2.14 \\
(2.06 / 2.24)\end{array}$ & $\begin{array}{c}40.7 \\
(31.3 / 53.1)\end{array}$ & $\begin{array}{c}9.5 \\
(7.0 / 12.9)\end{array}$ & $\begin{array}{c}54.7 \\
(59.3 / 48.8)\end{array}$ & $\begin{array}{c}3.3 \\
(4.4 / 2.0)\end{array}$ \\
\hline $\begin{array}{l}\text { Förder-/Hauptschul- } \\
\text { abschluss: } \\
\text { kein Wechsel }\end{array}$ & 3.20 & 2.07 & 36.2 & 11.4 & 53.6 & 3.7 \\
\hline $\begin{array}{l}\text { Förder-/Hauptschul- } \\
\text { abschluss: Absteiger }\end{array}$ & 3.12 & 2.07 & 40.2 & 15.2 & 61.8 & 9.2 \\
\hline $\begin{array}{l}\text { Förder-/Hauptschul- } \\
\text { abschluss: Aufsteiger }\end{array}$ & 3.20 & 2.14 & 34.6 & 13.2 & 56.1 & 5.3 \\
\hline $\begin{array}{l}\text { Realschulabschluss: } \\
\text { kein Wechsel }\end{array}$ & 3.19 & 2.06 & 39.6 & 7.8 & 57.0 & 2.8 \\
\hline $\begin{array}{l}\text { Realschulabschluss: } \\
\text { Absteiger }\end{array}$ & 3.19 & 2.07 & 42.9 & 10.4 & 66.5 & 8.1 \\
\hline $\begin{array}{l}\text { Realschulabschluss: } \\
\text { Aufsteiger }\end{array}$ & 3.18 & 2.12 & 41.9 & 9.0 & 58.1 & 3.1 \\
\hline $\begin{array}{l}\text { Abitur: } \\
\text { kein Wechsel }\end{array}$ & 3.27 & 2.04 & 36.5 & 4.1 & 47.1 & 2.2 \\
\hline Abitur: Aufsteiger & 3.13 & 2.15 & 42.8 & 8.1 & 50.2 & 2.7 \\
\hline
\end{tabular}

\subsection{Medienkonsum}

Im Rahmen der Befragung wurde der Medienkonsum auf verschiedene Weise erhoben. Da er sehr vom Geschlecht geprägt ist (vgl. Baier et al., 2010, S. 22ff), werden die nachfolgenden Auswertungen nur auf die männlichen Befragten bezogen. Die beiden zuerst dargestellten Variablen der Konsumzeit von Film/Fernsehen bzw. von Computerspielen wurden darüber erhoben, dass die Schüler für einen gewöhnlichen Schultag und zusätzlich für einen gewöhnlichen Wochenendtag angeben sollten, wie lange sie verschiedenen Tätigkeiten nachgehen. Die Zeit zum Schultag wurde mit fünf, die zum Wochenendtag mit zwei multipliziert; diese Werte wurden addiert und durch sieben geteilt, so dass die Durchschnittszeit für einen Tag ausgewiesen wird. Erkennbar ist, dass die Absteiger höhere Konsumzeiten aufweisen. Allerdings ist dieser Effekt weitestgehend dem Umstand geschuldet, dass mehr Absteiger Schulzweige besuchen, die zu 
niedrigeren Abschlüssen führen (und die Schüler in niedrigeren Abschlusszweigen längere Konsumzeiten aufweisen). Werden die Befragten innerhalb der drei unterschiedenen Abschlusszweige verglichen, stellen nicht die Absteiger, sondern die Aufsteiger eine eher besondere Gruppe dar, insofern sie ausgedehntere Konsumzeiten haben als Nicht-Wechsler. So sehen bspw. die Aufsteiger in den Abiturzweig 18 Minuten länger fern als die Nicht-Wechsler.

Daneben wurde der häufige Konsum von problematischen Medieninhalten erfragt. Der häufige Konsum von Gewaltfilmen umfasst dabei das mindestens wöchentliche Sehen von Horrorfilmen ab 16 oder 18 Jahren bzw. von Actionfilmen/Thrillern ab 18 Jahren. Der häufige Konsum von Gewaltspielen wurde über das mindestens wöchentliche Spielen von Ego/Third-Person-Shootern bzw. Beat-em-Ups/Prügelspielen abgebildet. Unterschiede sind dabei stärker bei den Gewaltfilmen festzustellen: Die Aufwie die Absteiger schauen häufiger entsprechende Filme. Dies gilt auch dann, wenn die Abschlusszweige separat betrachtet werden. Aufsteiger in den Abiturzweig weisen eine um ein Drittel erhöhte Rate häufiger Gewaltfilmkonsumenten auf (31.4 zu 23.2\%).

Zuletzt in Tabelle 8 wird der Anteil an Jugendlichen betrachtet, die Computerspielabhängig bzw. -abhängigkeitsgefährdet sind. Zur Messung der Computerspielabhängigkeit kam eine 14 Items umfassende Skala zum Einsatz (vgl. Rehbein, Kleimann \& Mößle, 2009), die u. a. nach dem Kontrollverlust oder Entzugserscheinungen fragt. Auch dabei gilt, dass sowohl Auf- als auch Absteiger öfter eine Abhängigkeit oder Gefährdung aufweisen. Im Vergleich der Abschlussgruppen zeigt sich allerdings, dass dies nur für die Schüler der Fall ist, die einen Förder-/Hauptschulabschluss bzw. die ein Abitur anstreben. Während bspw. 8.4\% der im Abiturzweig unterrichteten Aufsteiger abhängig oder gefährdet sind, sind es bei den Nicht-Wechslern nur 5.8\%. Es kann spekuliert werden, wie sich die erhöhten Raten erklären lassen. Möglicherweise müssen dabei für die Auf- und die Absteiger unterschiedliche Gründe benannt werden. Bei den Aufsteigern könnte es der Fall sein, dass sie den erhöhten schulischen Stress über das Spielen zu kompensieren versuchen. Bei den Absteigern hingegen könnte die Zuwendung zu Computerspielen damit in Zusammenhang stehen, dass sie sich außerschulische Bereiche erschließen, in denen sie Anerkennung erfahren können. 
Tabelle 8. Mediennutzung der Ab- und Aufsteiger, nur männliche $\mathrm{Be}-$ fragte (Mittelwerte bzw. in \%).

\begin{tabular}{|c|c|c|c|c|c|}
\hline & $\begin{array}{l}\text { Zeit Filme } \\
\text { schauen/ } \\
\text { Fernsehen }\end{array}$ & $\begin{array}{c}\text { Zeit Com- } \\
\text { puterspielen } \\
\text { (Online und } \\
\text { Offline) }\end{array}$ & $\begin{array}{c}\text { häufiger } \\
\text { Konsum } \\
\text { von Gewalt- } \\
\text { filmen }\end{array}$ & $\begin{array}{c}\text { häufiger } \\
\text { Konsum von } \\
\text { Gewalt- } \\
\text { spielen } \\
\end{array}$ & $\begin{array}{c}\text { Computer- } \\
\text { spielab- } \\
\text { hängig bzw. } \\
\text {-gefährdet }\end{array}$ \\
\hline kein Wechsel & $03: 25$ & $02: 18$ & 32.1 & 46.9 & 6.7 \\
\hline Absteiger & $03: 57$ & $02: 30$ & 42.7 & 49.7 & 9.6 \\
\hline Aufsteiger & $03: 36$ & $02: 23$ & 37.8 & 47.7 & 9.0 \\
\hline $\begin{array}{l}\text { Förder-/Hauptschul- } \\
\text { abschluss: kein Wechsel }\end{array}$ & $4: 10$ & $2: 34$ & 42.6 & 45.5 & 6.5 \\
\hline $\begin{array}{l}\text { Förder-/Hauptschul- } \\
\text { abschluss: Absteiger }\end{array}$ & $4: 16$ & $2: 36$ & 45.3 & 48.7 & 10.2 \\
\hline $\begin{array}{l}\text { Förder-/Hauptschul- } \\
\text { abschluss: Aufsteiger }\end{array}$ & 4:09 & $2: 55$ & 45.9 & 43.7 & 10.4 \\
\hline $\begin{array}{l}\text { Realschulabschluss: kein } \\
\text { Wechsel }\end{array}$ & $3: 33$ & $2: 24$ & 33.7 & 50.8 & 8.0 \\
\hline $\begin{array}{l}\text { Realschulabschluss: Ab- } \\
\text { steiger }\end{array}$ & $3: 29$ & $2: 20$ & 38.7 & 50.9 & 8.8 \\
\hline $\begin{array}{l}\text { Realschulabschluss: Auf- } \\
\text { steiger }\end{array}$ & $3: 44$ & $2: 25$ & 39.3 & 49.1 & 8.7 \\
\hline Abitur: kein Wechsel & $2: 45$ & $2: 02$ & 23.2 & 44.5 & 5.8 \\
\hline Abitur: Aufsteiger & $3: 03$ & $2: 01$ & 31.4 & 48.2 & 8.4 \\
\hline
\end{tabular}

\subsection{Multivariate Analysen}

Aus den bisher vorgestellten Analysen lassen sich Hinweise darauf entnehmen, welche Faktoren stärker und welche weniger stark mit dem Abbzw. Aufstieg in Verbindung stehen. In einer abschließenden multivariaten Auswertung sollen die wichtigsten Faktoren darauf hin geprüft werden, in welcher Beziehung sie unter Kontrolle der anderen Faktoren tatsächlich mit dem Ab- bzw. Aufstieg korrelieren. Hierzu wird auf binärlogistische Regressionsanalysen zurückgegriffen. Werte über 1 bedeuten, dass ein Faktor die Wahrscheinlichkeit der Zugehörigkeit zur Gruppe der Ab-/Aufsteiger erhöht, Werte unter 1, dass ein Faktor diese Wahrscheinlichkeit senkt. $\mathrm{Zu}$ beachten ist, dass die Regressionsanalyse i. d. R. zur Prüfung gerichteter Annahme und insofern zur Untersuchung von Einflussfaktoren herangezogen wird. Da weder mit der zugrundliegenden Querschnittsbefragung noch auf Basis theoretischer Überlegungen an die- 
ser Stelle gerichtete Annahmen geprüft werden, soll durch Einsatz der Regressionsanalysen einzig die Frage untersucht werden, hinsichtlich welcher Faktoren sich Auf-/Absteiger und Nicht-Wechsler unterscheiden. Was Ursache, was Wirkung ist, bleibt damit unbeantwortet.

Im ersten Modell in Tabelle 9 wird untersucht, hinsichtlich welcher Faktoren sich Absteiger und Nicht-Wechsler unterscheiden. Die Analysen wurden dabei sowohl für die Gesamtstichprobe als auch getrennt für Jungen und Mädchen gerechnet. ${ }^{5}$ Da Schüler, die ein Abitur anstreben, per se nicht abgestiegen sein können, wird der Abiturzweig in den Analysen nicht berücksichtigt. ${ }^{6}$ Nahezu für alle Faktoren ergeben sich signifikante Effekte. Es gilt daher, dass Absteiger im Vergleich zu Nicht-Wechslern

- seltener aus West- und Süddeutschland als aus Norddeutschland stammen;

- seltener im Realschulzweig und damit häufiger im Förder-/Hauptschulzweig zu finden sind;

- häufiger männlich sind; der Geschlechterunterschied wird also durch Einbezug der verschiedenen Faktoren nicht erklärt, warum also Jungen häufiger absteigen als Mädchen kann auf Basis der Faktoren nicht gesagt werden;

- Absteiger im Vergleich zu Nicht-Wechslern häufiger Schulschwänzer sind;

- häufiger Schulgewalt ausführen (gilt jedoch nur für die Jungen);

- seltener Schulangst gaben;

- häufiger nicht mit beiden leiblichen Elternteilen zusammenleben;

- häufiger Eltern mit mittlerem oder höherem Bildungsniveau haben;

5 Da sich die Richtung der Koeffizienten in den geschlechtergetrennten Modellen nicht von der Richtung der Effekte der Gesamtstichprobe unterscheidet, werden nur die Signifikanzen berichtet. In Modell II ergibt sich ein einziges Mal, dass ein Koeffizient für eine Geschlechtergruppe nicht der Richtung des Koeffizienten der Gesamtstichprobe entspricht (Schulgewalt Mädchen); dies ist mit einem Minus kenntlich gemacht, d. h. für Mädchen gibt es einen senkenden Effekt auf die Zugehörigkeit zur Gruppe der Aufsteiger.

6 Die Auswahl der einbezogenen Faktoren richtet sich danach, ob sich in den vorangegangenen bivariaten Analysen substanzielle Unterschiede in Bezug auf die betrachteten Gruppen gezeigt haben. Einige Faktoren, für die sich stärkere Unterschiede gezeigt haben, werden jedoch nicht in die multivariate Analyse einbezogen, weil nur Informationen für einen Teil aller Befragten zur Verfügung stehen. Die Computerspielabhängigkeit oder die Depressions-/Angstbehandlung wurden nur bei jedem dritten Schüler erfragt. Würden diese Faktoren hier einbezogen, würden die Fallzahlen zu stark sinken. 
- häufiger elterliche Gewalt in der Kindheit erfahren haben (gilt wiederum nur für die Jungen);

- häufiger eine ADHS-Diagnose berichten (nur Jungen), häufiger einen Selbstmordversuch unternommen haben (nur Mädchen), häufiger Rauschtrinken praktizieren und häufiger Cannabis konsumieren.

Absteiger sind damit in verschiedener Hinsicht eine belastete und auffällige Gruppe.

Werden die Ergebnisse zu Modell II, dass Nicht-Wechsler und Aufsteiger unterscheidet, betrachtet, lassen sich die Aufsteiger nicht in dieser Form charakterisieren. Zunächst zeigt sich, dass deutlich weniger Faktoren einen Beitrag dazu leisten, beide Gruppen zu unterscheiden. Es bestätigt sich, dass in Süd- und in Ostdeutschland signifikant häufiger als in Norddeutschland aufgestiegen wird. Zudem finden sich die Aufsteiger häufiger im Realschul- bzw. Abiturzweig. Es wird daneben der aus den bivariaten Auswertungen bekannte Befund bestätigt, dass männliche Jugendliche häufiger aufsteigen als weibliche Jugendliche. Damit kann mit den einbezogenen Faktoren erneut nicht der Geschlechtereffekt erklärt werden.

Zusätzlich gilt, dass Aufsteiger im Vergleich zu Nicht-Wechslern

- schlechtere Mathematiknoten berichten;

- eine höhere Schulangst haben;

- seltener aus Elternhäusern mit mittlerer oder höher Bildung kommen (gilt nur für Mädchen);

- etwas häufiger elterliche Gewalt in der Kindheit erlebt haben;

- häufiger Selbstmordversuche berichten (gilt nur in Bezug auf Jungen).

Mit der Ausnahme, dass aufsteigende Mädchen signifikant seltener Gewalt ausüben als Mädchen ohne Schulwechsel, ergeben sich sonst keine weiteren signifikanten Befunde. In beiden Modellen bleibt der Anteil erklärter Varianz zudem eher gering. Nur ca. 10\% der Varianz können erklärt werden. Weitere Faktoren, die Ab-/Aufsteiger und Nicht-Wechsler unterscheiden könnten, müssten in die Analysen einbezogen werden. 
Tabelle 9. Einflussfaktoren des Ab- und Aufstiegs (binär-logistische Regressionsanalysen; abgebildet: $\operatorname{Exp}(\mathrm{B})$ ).

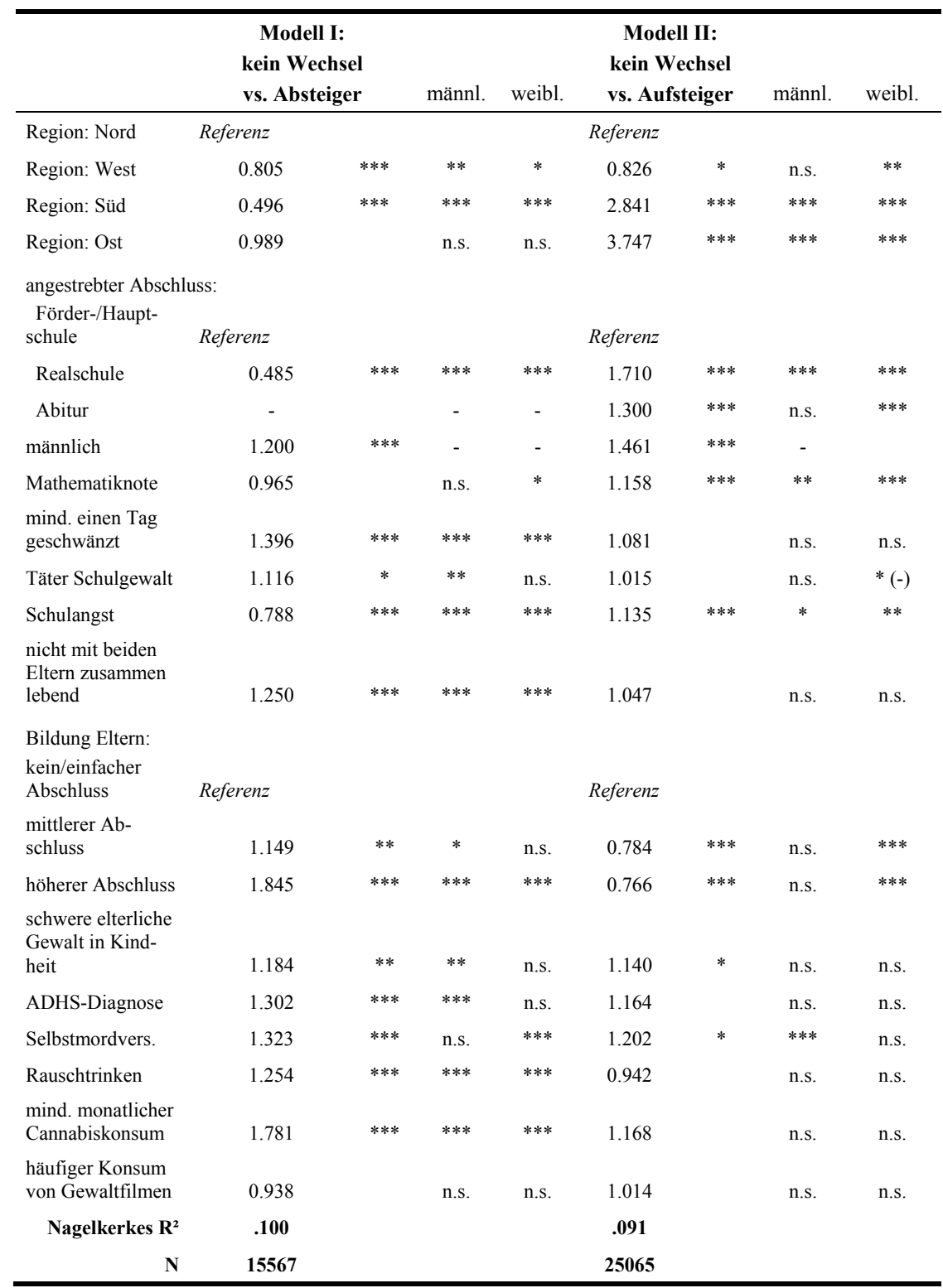

Anmerkung. $* p<.05, * * p<.01, * * * p<.001$, n.s. nicht signifikant bei $p<.05$. 


\section{Diskussion}

Bevor auf die zentralen Ergebnisse der vorgestellten Auswertungen eingegangen wird, ist zunächst noch einmal zu betonen, dass es sich hier um eine explorative Studie handelt. Dies ist einerseits deshalb der Fall, weil eine Befragung herangezogen wurde, die nicht eigens für die Untersuchung von Auf- und Abstiegsprozessen durchgeführt wurde. Zwar handelt es sich um eine deutschlandweit repräsentative Befragung mit sehr hoher Fallzahl, die Untersuchungsvariablen beschränken sich aber weitestgehend auf den Bereich des delinquenten Verhaltens. Zentrale individuelle Faktoren, die mit Auf- und Abstiegsprozessen in Verbindung stehen können, wurden nicht erfasst, was sich auch in den geringen erklärten Varianzen der multivariaten Modelle äußert. Darüber, welche Faktoren dies sind, kann an dieser Stelle nur spekuliert werden. Sicherlich müsste die bisherige Schullaufbahn bis in die Grundschule hinein betrachtet werden; und auch in Bezug auf das Elternhaus dürften noch weitere Faktoren von Relevanz sein (z. B. schulbezogenes Unterstützungsverhalten, Bildungsambitionen).

Andererseits ist der explorative Charakter der Studie darauf zurückzuführen, dass nur eine Querschnittsbefragung für die Auswertungen herangezogen werden konnte. Bei verschiedenen der vorgestellten Faktoren lässt sich nicht mit Sicherheit sagen, ob sie als Ursache oder als Folge des schulischen Auf- bzw. Abstiegs zu betrachten sind. Wenn sich bspw. zeigt, dass Absteiger häufiger Alkohol und Cannabis konsumieren, dann könnte dies das Resultat des Abstiegs sein, von der damit verbundenen Selbstwertschädigung oder von Anpassungsproblemen an das neue soziale Umfeld. Denkbar ist aber ebenso, dass diese Schüler bereits vor dem Abstieg eine höhere Risikobereitschaft zeigten, damit schon früher Alkohol und andere Drogen konsumierten und darüber ihr schulisches Leistungsvermögen gesenkt haben. Nur über Längsschnittstudien ließe sich die kausale Reihenfolge der Faktoren bestimmen.

Hinzuweisen ist an dieser Stelle zudem noch einmal darauf, dass sich die Auswertungen auf jene Schüler beschränken, die entweder nur einen Aufstieg oder nur einen Abstieg (bzw. keinen Wechsel) erlebt haben. Schüler, die sowohl einen Auf- als auch einen Abstieg erfahren haben oder die aufgrund anderer Gründe (z. B. Umzug) einen horizontalen Schulwechsel vollzogen haben, wurden aus den Analysen ausgeklammert. Dies ermöglicht zwar, dass eindeutig unterscheidbare Gruppen verglichen werden. Zugleich handelt es sich bei den ausgeklammerten Schülern sicher ebenfalls um interessante Fälle, deren Belastungen eine eingehendere 
Betrachtung verdienen. Dies unterstreicht, dass eine differenzierte, zusätzliche Faktoren integrierende Längsschnittstudie zu Schulwechseln notwendig ist.

Wenden wir uns den Befunden zu, so ist als erstes die Häufigkeit von Schulwechseln erstaunlich. In der neunten Jahrgangsstufe, der hier analysierten Altersgruppe, haben fast vier von zehn Schülern bereits irgendeinen Schulwechsel erlebt. Von den Schülern, die nur einer Wechselkategorie zuzuordnen sind (nur Auf- bzw. nur Abstieg), gaben etwas mehr Befragte einen Ab-als einen Aufstieg an. Immerhin jeder zehnte Jugendliche ist dementsprechend in seiner bisherigen Schullaufbahn schon einmal abgestiegen. Einbezogen wurden dabei auch Abstiege zwischen Schulzweigen in integrierten Schulformen.

Auf- und Absteiger können im Vergleich zu den Nicht-Wechslern als in verschiedener Hinsicht belastet oder auffällig eingestuft werden. Auch wenn dies die Befunde etwas verkürzt, lässt sich festhalten, dass Absteiger eher externalisierend, Aufsteiger eher internalisierend ihre Probleme verarbeiten. Unter erstgenannten werden fremdschädigende, unter letztgenannten selbstschädigende Verhaltensweisen gefasst (vgl. Fend, 2001, S. 417). So sind Absteiger häufiger Schulschwänzer, Schulgewalttäter und Alkohol- und Drogenkonsumenten. Bei Aufsteigern ergeben sich höhere Werte bei der Schulängstlichkeit und bei Selbstmordgedanken bzw. -versuchen. Zwar findet sich auch bei Absteigern, dass sie häufiger Selbstmordversuche unternehmen. Sie befinden sich aber häufiger als Aufsteiger bereits in Behandlung (z. B. wegen Angst oder Depression), d. h. deren Probleme sind häufiger bereits offenkundig und wurden einer Bearbeitung zugeführt. Bei Aufsteigern sind die Probleme hingegen häufiger verdeckt, angemessene Hilfe wird anscheinend seltener gewährt. Den psychischen Problemlagen der Aufsteiger sollte sich daher in den Schulen stärker als bislang gewidmet werden, so eine praktische Folgerung aus den Auswertungen.

Wenden wir uns den Korrelaten des Auf- und Abstiegs zu, so kann auf Basis der Ergebnisse zwischen strukturellen und individuellen Faktoren unterschieden werden. Bei den strukturellen Faktoren sind drei Faktoren $\mathrm{zu}$ benennen. Erstens variiert die Wahrscheinlichkeit des Schulwechsels mit der Region. Süd- und Norddeutschland bilden hier zwei Gegensätze: In Süddeutschland wird seltener ab- und häufiger aufgestiegen. Dies bedeutet allerdings nicht, dass das süddeutsche Schulsystem zu mehr sozialer Gerechtigkeit führen würde. Im Gegenteil: Dieses System ist alles in allem ungerechter als das norddeutsche System. Aufgrund der zum Zeitpunkt der Befragung in Bayern und Baden-Württemberg noch geltenden 
verbindlichen Laufbahnempfehlung besuchen zunächst noch weniger Jugendliche höhere Schullaufbahnen, obwohl es ihr schulisches Leistungsvermögen letztlich ermöglichen würde. In Norddeutschland ist die Laufbahnempfehlung unverbindlich; Eltern setzen sich häufiger darüber hinweg und schicken ihre Kinder auf höhere Schulformen. Dies erhöht das Risiko, dass die Jugendlichen hier scheitern. De facto passiert dies auch, so dass es häufiger zu Abstiegen kommt. In Süddeutschland hingegen sind die Schüler häufiger unterfordert im zugewiesenen Schulniveau; sie steigen auf, was die höhere Aufstiegsrate erklärt. Trotz des häufigeren Abstiegs in Norddeutschland und des häufigeren Aufstiegs in Süddeutschland eröffnet das Schulsystem in Norddeutschland den Schülern mehr Chancen, was am Beispiel des Abiturzweigs belegt werden kann: Während in Norddeutschland $36.0 \%$ aller Schüler ein Abitur ablegen werden, sind es in Süddeutschland nur 30.9\%, obwohl die Aufsteigerrate in den Abiturzweig 1.6-mal so hoch liegt wie in Norddeutschland. Einen Förder-/Hauptschulabschluss werden im Norden nur $24.8 \%$ ablegen, im Süden hingegen $39.8 \%$, obwohl im Norden mehr Schüler in diesen Zweig absteigen. Ohne die Verteilung der Schüler über die Schulformen zu betrachten sagen damit reine Auf- und Abstiegsraten nichts darüber aus, welche Bildungschancen ein Schulsystem einräumt. Norddeutschland schneidet dabei letztlich besser $a b$.

Ein zweiter struktureller Faktor ist die Schichtzugehörigkeit, in den Analysen operationalisiert über das Bildungsniveau im Elternhaus. Schüler aus gebildeteren Elternhäusern und damit höheren Sozialschichten steigen häufiger ab und seltener auf. Dies erscheint auch vor den eingangs zitierten Forschungsbefunden zunächst paradox. Eine Erklärung ist allerdings plausibel: Kinder aus gebildeteren Elternhäusern werden häufiger für den Besuch höherer Schulformen empfohlen. Lehrkräfte machen ihre Laufbahnempfehlung nicht einzig vom Leistungsniveau abhängig, sondern auch von anderen Faktoren, so bspw. der Annahme darüber, wie intensiv ein Kind von den Eltern schulisch unterstützt wird. Höhere Schichten investieren stärker in die Ausbildung der Kinder, weshalb die Empfehlung auf eine höhere Schulform auch bei schlechterem Notendurchschnitt legitim erscheint. Dies setzt diese Kinder einem höheren Risiko des Abstiegs aus; aufsteigen können sie zugleich weniger, weil sie schon den höheren Schulformen zugewiesen werden.

Der dritte strukturelle Faktor ist die Schulzweigzugehörigkeit. Die Analysen zeigen, dass die Auf- und Abstiegswahrscheinlichkeit nicht gleich über die Zweige verteilt sind. Abstiege in den Förder-/Hauptschulzweig sind demnach wahrscheinlicher als Abstiege in den Realschulzweig. Auf- 
stiege in den Hauptschulzweig sind weniger wahrscheinlich als Aufstiege in den Real- und Abiturzweig. Niedrigere Schulformen nehmen also häufiger Absteiger auf, die Chancen auf einen Aufstieg sind aber besonders gering. Niedrigere Schulformen $\mathrm{zu}$ verlassen ist demnach besonders schwer. Ob dies tatsächlich auf das geringe Leistungsniveau der Schüler zurückgeführt werden kann oder ob andere Barrieren entscheidend sind (z. B. geringe Bereitschaft des Realschulzweigs, solche Aufsteiger aufzunehmen; geringe Bereitschaft der Förder- und Hauptschullehrerschaft, Schüler für den Aufstieg zu empfehlen), lässt sich bislang nicht sagen.

Bezüglich der individuellen Faktoren soll auf zwei Faktoren eingegangen werden, da bezüglich der meisten betrachteten Faktoren eine Unsicherheit dahingehend besteht, ob es sich um Bedingungsfaktoren oder eher Folgen handelt. Hervorzuheben ist, dass das Geschlecht in signifikanter Weise mit dem Auf- wie dem Abstieg in Beziehung steht: Männliche Jugendliche steigen sowohl häufiger auf als auch häufiger ab. Der Geschlechterunterschied lässt sich mit den in die Analysen einbezogenen Variablen nicht erklären. Zudem ergeben sich hinsichtlich des Einflusses der verschiedenen betrachteten Variablen zum Teil Geschlechterunterschiede. Die Erklärung des doppelten Einflusses des männlichen Geschlechts fällt schwer. In jedem Fall gilt, dass männliche Jugendliche anscheinend häufiger als weibliche Jugendliche nicht ihrem tatsächlichen Leistungsvermögen entsprechend Schulzweigen zugewiesen werden. Dies muss nicht bedeuten, dass die Lehrkräfte bei der Laufbahnempfehlung oder die Eltern bei der Schulauswahl häufiger falsch liegen. $\mathrm{Zu}$ erinnern ist an dieser Stelle, dass Neuntklässler betrachtet werden, d. h. Jugendliche in der Hochphase der Pubertät. Möglicherweise zeigen sich bei männlichen Jugendlichen in dieser Phase stärkere, die Leistungsfähigkeit beeinflussende Veränderungen. Manche Jungen fallen besonders stark $a b$, andere hingegen holen besonders schnell auf. Die Varianz der Veränderung in der Pubertätsphase könnte bei Jungen höher sein als bei Mädchen, mit stärkeren Effekten (in die positive wie die negative Richtung) auf die schulische Leistungsfähigkeit. In jedem Fall sollten sich zukünftige Studien dem Geschlechtereffekt widmen und dabei soziale ebenso wie biologische Erklärungen prüfen.

Ein zweiter individueller Bereich an Faktoren betrifft die Familie. Nicht mit beiden leiblichen Elternteilen zusammenzuleben ist ein Risikofaktor für den Abstieg, Gewalt durch die Eltern zu erfahren für den Ab- wie den Aufstieg. Diese Befunde unterstreichen zunächst den Stellenwert der Familie für Schulwechselprozesse, der sich sicherlich auch in anderen Dimensionen zeigen würde, die hier aber aus Mangel an Indikatoren nicht 
abgebildet werden können. Familienauflösungen scheinen, bei aller Vorsicht bzgl. kausaler Aussagen, für Jugendliche stressauslösende Ereignisse zu sein, die sich negativ in den Schulleistungen niederschlagen und damit letztlich einen Abstieg bedingen können. Für Schulen ist dieser Befund von Bedeutung: Kinder und Jugendliche, die eine Trennung der Eltern erleben, bedürfen eine gesonderten Aufmerksamkeit. Ihr Abstieg ließe sich dann möglicherweise verhindern. Dass elterliche Gewalt ein Risikofaktor für den Abstieg ist, überrascht nicht, insofern dieses Erziehungsverhalten auch mit zahlreichen anderen negativen Phänomenen in Beziehung steht (vgl. u. a. Baier, Pfeiffer \& Thoben, 2013). Elterliche Gewalt ist ein Ausdruck davon, dass in den Familien eine verringerte Bindung an gesellschaftliche Normen und Werte besteht. Dadurch reduziert sich die Bindung der Kinder an zentrale gesellschaftliche Institutionen wie die Schule; Schulerfolg, schulische Anstrengung usw. sind für diese Kinder keine erstrebenswerten Ziele. Interessant ist, dass sich daneben auch ein etwas schwächerer Effekt der elterlichen Gewalt auf den Schulaufstieg ergibt, auch unter Kontrolle der Schichtzugehörigkeit. Dies lässt mehrere Deutungen zu: Möglicherweise setzen manche Eltern (leichte) Gewalt ein, um ihre Kinder zu besseren Leistungen anzutreiben. Die Angst vor Strafen erhöht das schulische Engagement, mit der Folge eines Aufstiegs. Denkbar ist daneben, dass Kinder, die Gewalt erfahren, sich in besonderer Weise der Schule zuwenden. Hier erhalten sie die Bestätigung, die sie zu Hause nicht erfahren; hier stoßen sie auf Personen (Lehrer), die ihnen Anerkennung entgegen bringen. Die stärkere Identifikation erhöht die Leistungsbereitschaft, was wiederum den Aufstieg wahrscheinlicher macht.

Bei verschiedenen hier geäußerten Deutungen für empirisch gefundene Zusammenhänge handelt es sich um vorsichtige Spekulationen. Sicherlich wären das ein oder andere Mal auch andere Deutungen denkbar. Die Untersuchung des Auf- und Abstiegs hat aber zu interessanten Befunden geführt; es handelt sich um ein wichtiges Forschungsfeld. Es wäre daher zu wünschen, dass sich in Zukunft noch stärker mit den strukturellen wie individuellen Faktoren des Auf- und Abstiegs (und sonstigen Schulwechseln) sowie der Beschreibung der Schüler, die diese Schulwechsel durchlaufen, beschäftigt wird. 
Wer sind die Aufsteiger, wer die Absteiger im deutschen Bildungssystem?

\section{Literatur}

Baier, D., Pfeiffer, C., Rabold, S., Simonson, J. \& Kappes, C. (2010). Kinder und Jugendliche in Deutschland: Gewalterfahrungen, Integration, Medienkonsum. Zweiter Bericht zum gemeinsamen Forschungsprojekt des Bundesministerium des Innern und des KFN (Forschungsbericht No. 109). Hannover: Kriminologisches Forschungsinstitut Niedersachsen.

Baier, D., Pfeiffer, C., Simonson, J. \& Rabold, S. (2009). Jugendliche in Deutschland als Opfer und Täter von Gewalt. Erster Forschungsbericht zum gemeinsamen Forschungsprojekt des Bundesministerium des Innern und des KFN (Forschungsbericht No. 107). Hannover: Kriminologisches Forschungsinstitut Niedersachsen.

Baier, D., Pfeiffer, C. \& Thoben, D. F. (2013). Elterliche Erziehung in Deutschland: Entwicklungstrends und Auswirkungen auf Einstellungen und Verhaltensweisen. Unveröffentlichtes Manuskript.

Bellenberg, G. (2012). Schulformwechsel in Deutschland. Durchlässigkeit und Selektion in den 16 Schulsystemen der Bundesländer innerhalb der Sekundarstufe I. Verfügbar unter: news.bagkjs.de/media/raw/Studie_Bertelsmann_Stiftung_Schulformwechsel_in_Deutschland.pdf [22.07.2014].

Farrington, D. P. (2010). Family Influences on Delinquency. In D. W. Springer \& A. R. Roberts (Hrsg.), Juvenile Justice and Delinquency (S. 203-222). Sudbury: Jones and Bartlett.

Fend, H. (2001). Entwicklungspsychologie des Jugendalters. Wiesbaden: VS Verlag.

Glaesser, J. \& Lauterbach, W. (2006). Prädiziert die Schulform auch den Schulabschluss? Oder: Auf- und Absteiger aus schulischen Laufbahnen. In K.-S. Rehberg (Hrsg.), Soziale Ungleichheit, kulturelle Unterschiede. CD-ROM (S. 1741-1753). Frankfurt am Main: Campus.

Henz, U. (1997). Der Beitrag von Schulformwechseln zur Offenheit des allgemeinbildenden Schulsystems. Zeitschrift für Soziologie, 26, 53-69.

Liegmann, A. (2008). Schulformwechsel. Eine empirische Analyse der subjektiven Sicht von Schülerinnen und Schülern auf ein Selektionsereignis. Dissertation an der Universität Duisburg-Essen. Verfügbar unter: http://duepublico.uni-duisburgessen.de/servlets/ DocumentServlet?id=17036 [22.07.2014].

Rehbein, F., Kleimann, M. \& Mößle, T. (2009). Computerspielabhängigkeit im Kindes- und Jugendalter. Empirische Befunde zu Ursachen, Diagnostik und Komorbiditäten unter besonderer Berücksichtigung spielimmanenter Abhängigkeitsmerkmale (Forschungsbericht No. 108). Hannover: Kriminologisches Forschungsinstitut Niedersachsen.

Walper, S. (2009). Trennung und Scheidung - Folgen für die Kinder im Spiegel deutscher Forschung. In S. Höfling (Hrsg.), Interventions for the Best Interest of the Child in Family Law Procedures (S. 29-55). München: Hanns-Seidel-Stiftung. 
